\title{
Coda und zugleich Schlussakkord
}

Nichts ist monokausal. Und doch hatte der beschriebene Tatbestand, ohne Zweifel und sogar zentral, zu tun mit Kafkas Zusammentreffen mit Albert Einsteins Relativitätstheorie. Eine neue, radikale Moderne war damals angebrochen. Zeitgleich entstanden nicht nur Ludwig Wittgensteins neue Sprachphilosophie neben Freuds Psychoanalyse und dem später dominierenden Existentialismus schon bei Husserl, später dann bei Heidegger. Auch jenes junge Lyrikgenie, für das bereits um die Jahrhundertwende die gesamte Wiener Kulturelite schwärmte, der noch knabenhafte Hugo von Hofmannsthal, österreichischer Adel aus jüdischem Geblüt, hatte bereits vor dem symbolträchtigen Jahr 1900 ausgesprochen, dass nun zu gelten hatte: „Modern ist Paul Bourget und Buddha; das Zerschneiden von Atomen und das Ballspielen mit dem All; modern ist das Zergliedern einer Laune..." Bei Hofmannsthal stand bereits weltraumferne, kalte kennerische Distanz gegen vormalig kuhwarme Identifikation, eine doppelte und bald schon kubistische Sicht auf die Welt, wo vorher eine zwangsvereinigende malerische (oder eben: Erzählerische) Kopie „des Ganzen“ erwartet worden war. Jedenfalls keine „epische Totalität“ länger in einer transzendental „obdachlos“ gewordenen Welt, wie sie Georg Lukács ebenfalls noch im Jahr 1900 diagnostiziert hatte. Auch die berühmte Heisenberg`sche Formel wird später für immer festschreiben, daß es unmöglich sein würde, das ganze Bild

\footnotetext{
${ }^{1}$ Hugo von Hofmannsthal, Aufsatz über Gabriele d'Annuncio, in: Gesammelte Werke, XXXII, 101,1 f. Ich danke der Hofmannsthal-Gesellschaft für Hilfe beim Auffinden des Zitats und für die Angabe seines Ortes.
}

(C) Der/die Autor(en) 2021

B. Neumann, Umrisse einer Dritten Kultur im interdisziplinären Zusammenspiel zwischen Literatur und Naturwissenschaft, ELECTRISCHER PROMETHEUS. Umrisse einer Dritten Kultur im interdisziplinären Zusammenspiel zwischen Literatur und Naturwissenschaft, https://doi.org/10.1007/978-3-662-63204-8_22 
$\mathrm{zu}$ erhalten aus nur einer Perspektive, respektive Versuchsanordnung. Allüberall Koinzidenzen von Kunsttheorie und Physik, von Musik und Gesellschaftsentwicklung. Ein Triumph des Transdisziplinären als eines Hauptelements in einer möglichen „Dritten Kultur“, mit Hofmannsthals zitierter, berühmt gewordener Ansage als kanonischer Formel? Der Große Krieg führte 1918 jedenfalls zur Liquidation der vormaligen, wie immer bereits hoch strapazierte Harmonie im Zusammenleben der Ethnien und Nationen innerhalb der altösterreichischen Monarchie: Finis austriae als finis mundi. Und zugleich, geradezu kompensatorisch, zur Entstehung eines ganz neuen Roman-Musters bei Franz Kafka als Beginn einer neuen Erzähl-Welt, eben der des 20. und bereits 21. Jahrhunderts. Doch war es nicht Hofmannsthal, sondern der Mann aus Prag, und eben auch nicht Rilke aus der gleichen Moldaumetropole, der der geniale literarische Ballspieler mit der neuesten Realität werden sollte. Es war Franz Kafka. Nur er hatte Einstein getroffen. Gewiss, auch Brod war dabei gewesen, doch der schrieb andere Literatur. Alles aber hatte zu tun mit der „magischen“ Qualität der Stadt Prag als einer der Astronomen und der Alchimisten, der Musiker und der Mystiker, der Macht und der Wissenschaft, hier vereint von alters her. „Einstein in Prag" brachte beide Elemente zusammen. Bewirkte auf diese Weise die Kernfusion, die ihrerseits eine neue Romanwelt, die des Franz Kafka, hervorbringen sollte. Sie geriet zum bis heute überzeugendsten Gegenentwurf zu Keplers Harmonia Mundi als der ebenfalls „wissenschaftlichen“ Beschwörung einer von der klassischen Antike herstammenden sinnvoll-geschlossenen Welt. Mithin noch immer Kafka als Werfels „Neutöner“, der eine obsolet gewordene Harmonik außer Kurs setzte. Womit sich der Kreis schließt. Aber gewiss nicht als Kurzschluss. Zum Abschluss noch einmal: Auftritt Theodor Wiesengrund Adornos. Der negativ dialektische Denker hatte bereits 1955 die folgenden Sätze drucken lassen (und darin einen Befund ausgesprochen, der bis heute aktuell erscheint): „Die Beliebtheit Kafkas, das Behagen am Unbehaglichen, das ihn zum Auskunftsbüro der je nachdem ewigen oder heutigen Situation des Menschen erniedrigt, ... weckt Widerwillen dagegen, mitzutun... Aber gerade der falsche Ruhm, ... zwingt zur Insistenz vor dem Rätsel... “ Und weiter: ,Jeder Satz steht buchstäblich, und jeder bedeutet. Beides ist nicht, wie das Symbol es möchte, verschmolzen, sondern klafft auseinander, und aus dem Abgrund dazwischen blendet der grelle Strahl der Faszination."2 So war es; so ist es. Wo Adorno einen eher verschwiemelten Existentialismus kritisierte (,der Mensch ist

\footnotetext{
${ }^{2}$ Theodor W. Adorno, Aufzeichnungen zu Kafka, a. a. O., S. $302 \mathrm{ff}$.
} 
ins Nichts gehalten“), könnte man zeitgenössisch die jüngst verstorbene „French Theory" ins Spiel bringen: Derrida legte sich den Text von Vor dem Gesetz, so zurecht, dass er gelehrt zum für Kafkas Text belanglosen, doch gut klingenden „Olfaktorischen“ zu gelangen vermochte, wie geschildert vor versammelter internationaler Presse in Londons „Royal Society“, also dort, wo einst Charles Darwin seine Evolutionslehre entwickelt hatte. Welch „electrisierender“" Vorgang!

Die abschliessende Coda lautet, bestimmt von für sie (als musikalische Figur untypischer) Ironie: In der Moldau-Stadt Prag finden sich selbstverständlich vielerlei Spuren vor allem von Kafka, aber auch von Albert Einstein. Franz Kafka, zum Touristenmagnet mit weltweiter Ausstrahlung avanciert, besitzt seine Denkmäler, Gedenkplaketten, Archive und Museen. Schließlich hat dieser stille Nachtschreiber die Weltliteratur unwiderruflich verändert. Wie das mit Einsteins Aufenthalt in der magischen Stadt zusammenhing, wurde dargestellt. Es ist nicht ohne Ironie, deshalb ein geeigneter Abschluss, dass es ausgerechnet Prags Touristenindustrie gelungen ist, was weder in der Einstein-, noch in der KafkaForschung bislang „wirklich“ Ereignis wurde: Die beiden Jahrhundert-Genies zusammenzubringen. In der Lesnická ulice 7, nicht weit von der Moldau, und am Ort von Einsteins wissenschaftlichem Wirken in Prag in den Jahren 1911/12 schaut ein betagter (also bereits mit dem Nobelpreis gesalbter?) Albert-EinsteinKopf nachdenklich von der Wand. Die Inschrift lautet: „Here lived and worked in the years 1911 and 1912 Albert Einstein.“ Doch die Erinnerungsplakette „depicts a very aged Einstein, much too old to relate to the historical moment it describes, and the text narrates the connections Einstein made in this house with Franz Kafka."3 So weit, so nüchtern-richtig. Doch wie Mozart sein Salzburger Gartenhäuschen, so hat auch Einstein seine Vereinigung mit Kafka eher als Touristengag erhalten. Es ging um jemanden, an den er sich im Leben nicht mehr so recht zu erinnern vermochte - dessen Schloss er aber dennoch gelesen und, man erinnert sich, für „pervers“ befunden hatte. Im Einstein-Jubiläumsjahr 1979 geschah die Anbringung von Albert Einsteins beschriebenem Kopf, da war auch bereits sein Gehirn, wider den testamentarischen Willen des Genies, in Scheibchen geschnitten zur „Wissenschafts“-Attraktion gemacht worden. In diesem Jubiläumsjahr erschienen übrigens noch vereinzelt Artikel über Einstein und Kafka, während am Old Town Square der Stadt Prag, dort wo einst Berta Fanta ihren Salon abhielt, tatsächlich Ereignis wurde, was auch dieses Buch

\footnotetext{
${ }^{3}$ Michael D. Gordin, Einstein in Bohemia, a. a. O., S. 265.
} 
beschwört: Eine offizielle Inschrift, auf Englisch, erinnert daran, dass Kafka und Einstein in diesen Räumen zusammengetroffen waren. Und dennoch geschah dieses Zusammentreffen nicht im realen Prag des heutigen Tourismus; sondern in der Stadt der Brahe und Kepler, der Meyrink, Rilke und Kundera, also in der „goldenen Stadt“ einer immer schon existierenden „Dritten Kultur“. „Bohemia has long since vanished, at least from the real cartography of the world. It has moved instead to the land of myth. There you can also find Einstein" ${ }^{* 4}$ - und dort findet man, seinerseits unsterblich, Franz Kafka vor, als den „electrischen Prometheus" unserer Moderne, des mythischen Prag so ganz heutigen Großmeister aller modern erzählenden Literatur.

${ }^{4}$ Michael D. Gordin, Einstein in Bohemia, a. a. O., S. 265. 
Open Access Dieses Kapitel wird unter der Creative Commons Namensnennung 4.0 International Lizenz (http://creativecommons.org/licenses/by/4.0/deed.de) veröffentlicht, welche die Nutzung, Vervielfältigung, Bearbeitung, Verbreitung und Wiedergabe in jeglichem Medium und Format erlaubt, sofern Sie den/die ursprünglichen Autor(en) und die Quelle ordnungsgemäß nennen, einen Link zur Creative Commons Lizenz beifügen und angeben, ob Änderungen vorgenommen wurden.

Die in diesem Kapitel enthaltenen Bilder und sonstiges Drittmaterial unterliegen ebenfalls der genannten Creative Commons Lizenz, sofern sich aus der Abbildungslegende nichts anderes ergibt. Sofern das betreffende Material nicht unter der genannten Creative Commons Lizenz steht und die betreffende Handlung nicht nach gesetzlichen Vorschriften erlaubt ist, ist für die oben aufgeführten Weiterverwendungen des Materials die Einwilligung des jeweiligen Rechteinhabers einzuholen.

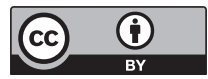

